\title{
Energy-Aware Base Stations: The Effect of Planning, Management, and Femto Layers
}

\author{
G. Koutitas, ${ }^{1,2}$ L. Chiaraviglio, ${ }^{3}$ Delia Ciullo, ${ }^{4}$ M. Meo, ${ }^{5}$ and L. Tassiulas ${ }^{1}$ \\ ${ }^{1}$ Computer Engineering and Telecommunications, University of Thessaly, 38221 Volos, Greece \\ ${ }^{2}$ School of Science and Technology, International Hellenic University, Thermi, Greece \\ ${ }^{3}$ DIET Department, University of Roma-La Sapienza, Via Eudossiana 18, Rome, Italy \\ ${ }^{4}$ Mobile Communications Department, Eurecom, Sophia Antipolis, 450 Route des Chappes, 06410 Biot, France \\ ${ }^{5}$ Telecommunication Networks Group, Politecnico di Torino, Corso Duca degli Abruzzi 24, 10129 Torino, Italy
}

Correspondence should be addressed to G. Koutitas; george.koutitas@gmail.com

Received 21 October 2013; Accepted 3 January 2014; Published 30 March 2014

Academic Editor: Vincenzo Eramo

Copyright (c) 2014 G. Koutitas et al. This is an open access article distributed under the Creative Commons Attribution License, which permits unrestricted use, distribution, and reproduction in any medium, provided the original work is properly cited.

\begin{abstract}
We compare the performance of three base station management schemes on three different network topologies. In addition, we explore the effect of offloading traffic to heterogeneous femtocell layer upon energy savings taking into account the increase of base station switch-off time intervals. Fairness between mobile operator and femtocell owners is maintained since current femtocell technologies present flat power consumption curves with respect to served traffic. We model two different user-to-femtocell association rules in order to capture realistic and maximum gains from the heterogeneous network. To provide accurate findings and a holistic overview of the techniques, we explore a real urban district where channel estimations and power control are modeled using deterministic algorithms. Finally, we explore energy efficiency metrics that capture savings in the mobile network operator, the required watts per user and watts per bitrate. It is found that the newly established pseudo distributed management scheme is the most preferable solution for practical implementations and together with the femotcell layer the network can handle dynamic load control that is regarded as the basic element of future demand response programs.
\end{abstract}

\section{Introduction}

The traffic proportional power consumption of information and communication technology (ICT) equipment is regarded as a promising technological growth for the improvement of the overall energy efficiency of the sector [1]. Despite the fact that individual components are already operating in different power states, according to the input load, the aggregated power profile of the network presents low correlation with traffic. This results in a power waste and a low efficiency, especially during off-peak hours. In a cellular network, base stations (BSs) are regarded as the weakest part in terms of energy efficiency since they comprise network critical physical infrastructure (NCPI) that introduces high no load losses (i.e., the losses that are associated with cooling and power units, which do not depend on the traffic) [2]. Recent 3 Generation Partnership Program (3GPP) regulations and new standards indicate the required steps for traffic proportional mobile network power consumption $[3,4]$ that incorporate BS management schemes and radio network planning phases $[5,6]$. Depending on the network layout and the traffic pattern, it has been shown that significant savings can be achieved by reducing the number of active BSs by introducing sleep modes [7].

This paper aggregates and simulates the most important BS management schemes found in the literature on three different network topologies that were derived by an optimization algorithm. The BS management schemes are the centralized, the distributed, and the pseudo distributed schemes, each one presenting crucial differences in terms of savings and complexity. The network topologies were derived by the minimum transmitter, the minimum power consumption, and a hybrid planning strategy that are regarded as the most dominant planning strategies for future networks. 
In addition, the paper explores the effect of heterogeneous networks (HetNets), such as femtocell, on energy savings and dynamic load control.

It is shown that the hybrid planning strategy coupled together with the pseudo distributed BS management scheme and also taking advantage of the HetNet can provide not only great OPEX reductions to the mobile network operator but also dynamic load control that is regarded as a fundamental element of future demand response programs. Basic capital expenditure (CAPEX) and operational expenses (OPEX) computations are performed.

The paper is organized as follows. In Section 2 a description of the related work is presented. Section 3 presents the network model used together with the power control scheme, the femto user association rules, and the propagation models. Section 4 presents the network architecture and the concept of critical and flexible BSs. Section 5 presents the BS management algorithms and Section 6 presents the used energy efficiency metrics. Finally, Section 7 explores the simulation results and Section 8 concludes our work.

\section{Related Work on Energy Efficiency}

A centralized BS management scheme for a macro/microarchitecture is presented in [7]. In [8] the authors examine centralized management algorithms based on the least load and cell overlap criteria, applied upon a real network configuration of central London. The concept of energy partitions for Self-Optimized Network (SON) operation is given in $[9,10]$. In [9] a pseudo distributed control scheme is introduced and applied upon a hexagonal network configuration. The partition in that case is assumed to comprise the flexible stations under the administrative domain of the critical ones. In $[11,12]$ authors investigate traffic proportional network power consumption for $3 \mathrm{G}$ networks utilizing optimization algorithms. In [13] centralized and distributed algorithms are used for a microarchitecture. A different approach to energy management is given in $[14,15]$ presenting the case of cooperation between two mobile operators. In [16] authors examine the BS planning effect showing that micro-BS architectures can increase the network's performance. Similar observations are also derived in [17]. In addition, the femtocell layer is shown to be of minor importance if one considers the network in large scale and does not impose any BS management schemes. In [18] the authors prove that a microcell based network provides the important gains, but the network does not present traffic proportional characteristics. In [19] the effect of service rate on the energy consumption of mobile network that supports switch-on/-off schemes is analyzed. Physical layer energy efficient techniques are presented in [20] focusing on physical layer and power control techniques. Finally, BS power consumption models that can be used for the estimation of the network's energy demand are investigated in [21-23]. A quantitative analysis of the effect of femtocell layer (HetNet) on a theoretical hexagonal network configuration is presented in $[24,25]$. The authors examine CAPEX and OPEX characteristics according to femtocell deployment densities in a macrofemto architecture. In this paper we extend the investigation of the effect of three different planning and management procedures on a real network configuration and we explore the feasibility of a pseudo distributed management algorithm together with the effect of the femtocell layer on energy efficiency and savings. We perform simulations for two different user-femtocell association rules to capture realistic and theoretically maximum gains derived from the HetNet. Finally, we explore various energy efficiency metrics to capture not only the energy savings at the operator side but also the energy efficiency of offered service. The paper gives a more holistic view compared to the available literature in the area of network planning, BS management, and offloading for energy efficiency.

\section{The Cellular Network}

3.1. Description of Scenario. An area $A$ of central London is under investigation and is presented in [18]. The area is a central urban district modeled by a vector map. The vector map describes the facets of the buildings that are incorporated in the ray tracing algorithm for channel estimation. It is a rectangular area of size $1.8 \mathrm{Km} \times 1.8 \mathrm{Km}$ with equally spaced points of distance $50 \mathrm{~m}$ creating a set of possible coordinates CRD. We partition the coordinates in two different sets, that is, the outdoor $\mathrm{CRD}_{0}$ and indoor coordinates $\mathrm{CRD}_{\mathrm{I}}$ with $\mathrm{CRD}_{0} \cap \mathrm{CRD}_{\mathrm{I}}=\varnothing$ and $\mathrm{CRD}_{0} \cup \mathrm{CRD}_{\mathrm{I}}=\mathrm{CRD}$. Within $A$ there is a set of sites $S$, with identifiers $s_{j} \in S, j \in\left\{1,2, \ldots N_{\mathrm{Tx}}\right\}$ where $N_{\text {Tx }}$ is the number of BSs. These sites are macrocell and microcell BSs that are under the administrative domain of the mobile operator. Furthermore, we assume that there are opportunistically deployed femtocell access points (FAPs) in the network within the users' premises. These stations form a set $f \in F, f \in\left\{1,2, \ldots N_{\mathrm{FTx}}\right\}$ where $N_{\mathrm{FTx}}$ is the number of FAPs.

We define as $M_{a} \subset S$ and $M_{i} \subset S$ the set of macrocells and microcells, respectively. Each site, $j \in S$, has 5 characteristic values, representing the position, the type of station, the maximum transmit power level, and the antenna gain following the notation in [18]. In a similar approach, the FAPs, $f \in F$, are characterized by the position which is defined by Cartesian coordinates $\left\{x_{f}, y_{f}\right\} \in \mathrm{CRD}_{\mathrm{I}}$, the height $H_{f}$, the maximum transmit power level $P_{f}=0.5 \mathrm{~W}$, and the antenna gain $G_{f}=2 \mathrm{dBi}$. Table 1 reports the main notation used in this work.

We model the users (described by a set $U$ ) and their properties as a population in the network topology using the user equivalent definition [8]. Each user is characterized by the location, the antenna gain, and the type of service requested indicating the required data rate and minimum signal to noise ratio threshold $\left(\mathrm{Eb} / \mathrm{Io}=\delta_{i}\right)$. It is assumed that the user positions, during one simulation, are constant with time and uniformly distributed over $\mathrm{CRD}_{o}$. Since the outdoor coordinates in the urban district are not uniform due to the building architecture, the uniform distribution of users over $\mathrm{CRD}_{o}$ yields more concentration in wide roads and 
TABLE 1: Main notation.

\begin{tabular}{|c|c|c|}
\hline Symbol & Explanation & Value \\
\hline$A$ & Area under investigation & $1.8 \times 1.8 \mathrm{Km}$ \\
\hline CRD & Set of possible coordinates & Equally spaced points of distance $50 \mathrm{~m}$ \\
\hline$S$ & Set of sites & Cartesian coordinates inside $A$ \\
\hline$M_{a}$ & Set of macrocells & 23 possible sites \\
\hline$M_{i}$ & Set of microcells & 43 possible sites \\
\hline$N_{\mathrm{Tx}}$ & Number of base stations (macrocells and microcells) & 69 \\
\hline$F$ & Set of femtocells access points & Cartesian coordinates inside $A$ \\
\hline$N_{\text {FTx }}$ & Number of femtocells access points & $25-75$ \\
\hline$\left\{x_{f}, y_{f}\right\}$ & Cartesian coordinates of femtocell $f$ & Coordinates inside $A$ \\
\hline$H_{f}$ & Height of femtocell $f$ & $1.5 \mathrm{~m}$ above floor \\
\hline$P_{f}$ & Maximum transmit power level for femtocell $f$ & $\{0,0.5\} \mathrm{W}$ \\
\hline$P_{j}$ & Maximum transmit power level for base station $j$ & $\begin{array}{l}\{0,10,20,30,40,50\} W \text { (macro) } \\
\{0,1,2,3,4,5\} W \text { (micro) }\end{array}$ \\
\hline$G_{f}$ & Antenna gain for femtocell $f$ & $2 \mathrm{dBi}$ \\
\hline$U$ & Set of users & Cartesian coordinates inside $A$, with $|U|=847$ \\
\hline$\delta_{i}$ & Signal to noise ratio threshold for user $i$ & $5 \mathrm{~dB}$ for voice, $2.5 \mathrm{~dB}$ for video, and $2 \mathrm{~dB}$ for web service \\
\hline$G(U, S)$ & Affinity graph for users and base stations & Set of edges between $U$ and $S$ \\
\hline$G(U, F)$ & Affinity graph for users and femtocells & Set of edges between $U$ and $F$ \\
\hline$B_{j}$ & Best server site & Identifier of the site \\
\hline$p_{j}^{c}$ & $\begin{array}{l}\text { Power associated with the control channel of base } \\
\text { station } j\end{array}$ & $15 \%$ of the total power \\
\hline $\mathrm{HN}_{j}$ & Soft handoff site & Identifier of the site \\
\hline$q$ & Soft handoff margin & $5 \mathrm{~dB}$ \\
\hline$l_{i j}$ & Path loss from user $i$ to base station $j$ & Variable \\
\hline $\mathrm{CV}_{j}$ & Coverage umbrella of base station $j$ & Variable \\
\hline$x_{i j}$ & $\begin{array}{l}\text { Variable indicating if user } i \text { is associated with base } \\
\text { station } j\end{array}$ & $\{0,1\}$ \\
\hline$G_{i}$ & Antenna gain of base station $i$ & $10 \mathrm{~dB}$ (macro), $3 \mathrm{~dB}$ (micro) \\
\hline$G_{i}$ & Antenna gain of user $i$ & $2 \mathrm{dBi}$ \\
\hline$\omega$ & Orthogonality factor & 0.6 \\
\hline$v_{i}$ & Voice activity & 0.58 \\
\hline$\mu_{i}$ & Carrier to interference plus noise ratio for user $i$ & Variable \\
\hline$\pi_{p}$ & Minimum sensitivity threshold & $-115 \mathrm{dBm}$ \\
\hline$n$ & Thermal noise & $-174 \mathrm{dBm} / \mathrm{Hz}$ \\
\hline $\bar{p}_{j}$ & Mean transmit power of base station $j$ & Variable \\
\hline$r_{i}$ & Required rate for user $i$ & $\begin{array}{l}12 \mathrm{Kbps} \text { for voice, } 64 \mathrm{Kbps} \text { for vide, } 144 \mathrm{Kbps} \text { for web } \\
\text { service }\end{array}$ \\
\hline C & Chip rate & $3.87 \times 10^{6} \mathrm{cps}$ \\
\hline$W_{j}$ & Total consumed power for base station $j$ & Equation (8a) \\
\hline$W_{f}^{F}$ & Total consumed power for femtocell $f$ & $10 \mathrm{~W}$ \\
\hline$R_{j}$ & Maximum data rate processed by base station $j$ & $14 \mathrm{Mbps}$ (macro), 4 Mbps (micro) \\
\hline$a_{j}$ & Binary variable indicating if base station $j$ is used & $\{0,1\}$ \\
\hline$U^{\max }$ & Set of users during peak hour & Cartesian coordinates inside $A$ \\
\hline$N^{\max }$ & Number of users during peak hour & 847 \\
\hline$N^{\min }$ & Minimum number of users & 40 \\
\hline$t$ & Current time & Variable \\
\hline$t_{\zeta}$ & Decision time & Variable \\
\hline$T$ & Maximum time & $24 \mathrm{~h}$ \\
\hline
\end{tabular}


TABle 1: Continued.

\begin{tabular}{lll}
\hline Symbol & Explanation & Value \\
\hline$\Omega_{k}$ & $\begin{array}{l}\text { Set of base stations that are controlled by a critical base } \\
\text { station } k \text { in the pseudo distributed algorithm }\end{array}$ & Variable \\
$\varepsilon$ & Load threshold & 0.3 for macros and 0.03 for micros \\
\hline
\end{tabular}

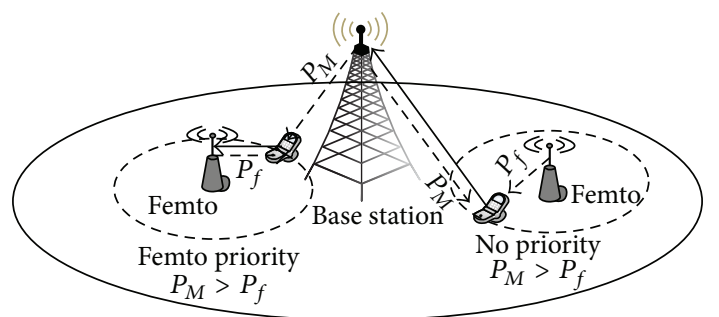

FIGURE 1: User association rules. Macro/micro architecture and femto access.

open parks, capturing real-life scenarios. A snapshot $S N$ is described by mapping each mobile user to a location in the area and the appropriate user characteristics. A bigraph, the affinity graph, is used to model the affinity of user equivalents to sites, $G(U, S)$ or $G(U, F)$, which is undirected with vertex set $V\{S \cup U\}$ or $V\{F \cup U\}$ such that there is an edge between users $u \in U$ and $s \in S$ or $f \in F$ if user $u$ is under the coverage umbrella of site $s$ or FAP $f$. A subgraph of the affinity graph, the association graph, represents the real association of users with the site based on the server selection criteria.

3.2. User Association Rules. It is assumed that the FAPs are in open loop access and thus users can be associated with the femtos according to capacity and signal constraints. We distinguish two cases (see Figure 1). In the first case, BSs and FAPs are treated in a balanced manner and the user is connected to $j \in S$ or $f \in F$ according to the best server criteria. This user association rule is named as no priority. In the second approach, users are assumed to prefer femtocell connection and are connected to a femto if and only if the received signal from the FAP satisfies quality of service (QoS) criteria. This user association rule is named as femto priority.

3.2.1. No Priority Rule. We define as the best server site $B_{j}$ the site $j \in S$ or $j \in F$ that associates users $i \in U$ with $B_{j}$ under the following criteria [26] $\left(l_{i j}<1\right.$ represents the path loss of user $i$ from site $j$ ):

$$
B_{j \in S \cup F}:=\{i\} \longrightarrow \underset{i}{\arg \max }\left(G_{j} \cdot g_{i} \cdot p_{j}^{c} \cdot l_{j i}\right) .
$$

In the above formulation $p_{j}^{c}=z \cdot P_{j}$ is the power associated with control channels and is a function of factor $z$ (usually it is $z=0.15$ describing $15 \%$ of radio frequency (RF) out power associated with control channels [26]). To model soft handoff mechanism, we define as the soft handoff site $\mathrm{HN}_{j}$ the site that associates $i \in U$ with $\mathrm{HN}_{j}$, under the following criteria ( $q$ is the soft handoff margin):

$$
\begin{gathered}
\mathrm{HN}_{j \in S \cup F}:=\left\{u_{i} \in U: \exists k \neq j \text { such that }\left|l_{i j} G_{j} p_{j}^{p}-l_{i k} G_{k} p_{k}^{p}\right|\right. \\
\leq q, q=5 \mathrm{~dB}\} .
\end{gathered}
$$

Thus, in the association graph, each vertex can have a degree of 1 or larger. By denoting $x_{i j}=1$ if user $i$ is connected to site $j$ and 0 otherwise, we impose the following constraints:

$$
\begin{aligned}
& \sum_{j \in S \cup F} x_{i j}=1, \quad \forall i \in B_{j}, \\
& \sum_{j \in S \cup F} x_{i j}>1, \quad \forall i \in \mathrm{HN}_{j} .
\end{aligned}
$$

The user is assumed to be within the coverage umbrella (set of users belonging to $\mathrm{CV}_{j}$ ) of a specific cell if and only if QoS criteria are satisfied. In a mathematical form it is

$$
\begin{aligned}
& \mathrm{CV}_{j} \\
& :=\left\{\begin{array}{c}
u_{i} \in B_{j}, \mathrm{HN}_{j}: l_{i j} G_{j} p_{j}^{c} \geq \pi_{p}, l_{j i}^{\uparrow} G_{i} p_{i}^{\uparrow} \geq \pi_{p}^{\uparrow}, \\
\gamma_{i j}=\frac{l_{i j} G_{j} p_{i j}}{n+\omega l_{i j} G_{j}\left(\bar{p}_{j}-v_{i} p_{i j}\right)+\sum_{k \in U \backslash H N_{j}, k \neq j} l_{i k} G_{k} \bar{p}_{k}} \geq \mu_{i}
\end{array}\right\},
\end{aligned}
$$

where $\omega$ is the orthogonality factor $(\sim 0.6), n$ is the thermal noise, $v_{i}$ is the voice activity $(\sim 0.58), \mu_{i}$ is the required carrier to interference plus noise ratio, $\pi_{p}$ is a minimum threshold [26], and $\bar{p}_{j}$ is the mean transmit power for base station $j$. Indicator $\uparrow$ symbolizes the uplink channel.

3.2.2. Femto Priority Rule. This rule gives priority to the FAPs. It is used to model the upper bound of energy savings that can be obtained by femtocells. Under this rule the users in the network are categorized in two sets. In the first set, $U^{F} \subset U$, the users are connected to FAPs if they receive adequate power from the FAPs; that is, $l_{i f} G_{f} p_{f}^{c} \geq \pi_{p}, f \in F$. To simplify computations, we assume that the FAPs and the macro-/micronetwork operate in different channels and thus interference between the multitier networks is negligible [27]. The remaining users are treated similar to the equations in (1), (2), and (4) taking into account only BSs $j \in S$.

3.3. Channel Estimation Algorithms. Due to the complex nature of the urban scenario, two different channel estimation algorithms were considered. A deterministic 3D ray tracing 
algorithm was used for field predictions between a user and a macro-/microstation and an empirical propagation model was used to model the channel gain between a FAP and a user. The empirical model was preferred since the indoor clutter within the houses is opportunistic and thus it cannot be deterministically modeled.

3.3.1. Ray Tracing Algorithm. The used ray tracing algorithm is presented in [18]. It is based on an intelligent preprocessing of the database algorithm where the vector data of the buildings is described by tiles and segments. A ray is propagated in the environment by multiple reflections, diffractions, or a combination of the above mechanisms between the tiles and segments, until it reaches the receiving point.

3.3.2. Empirical Algorithm for Femtos. To model the indoor to outdoor propagation, the Keenan-Motley formulation was used as presented in $[28,29]$. The propagation loss between a user and a FAP is

$$
\begin{aligned}
l^{\mathrm{dB}}= & 20 \log _{10}\left(\frac{4 \pi f}{c}\right)+20 \log _{10} d+q_{\mathrm{in}} W_{\mathrm{in}}+ \\
& +q_{\mathrm{ex}} W_{\mathrm{ex}}+F n^{((n+2) /(n+1)-0.46)} .
\end{aligned}
$$

All parameters are explicitly defined in [28]. For the purpose of the simulation results it was assumed that the random variables are uniformly distributed with $q_{\text {in }} \leftarrow U_{n}[1,10]$, $q_{\text {ex }} \leftarrow U_{n}[1,4], n \leftarrow U_{n}[1,5], W_{\text {in }}=5 \mathrm{~dB}, W_{\text {ex }}=7 \mathrm{~dB}$, $F=18 \mathrm{~dB}$, and $f=2 \mathrm{GHz}$.

3.4. Power Control Mechanism. The associated downlink power from BS with the served users is computed according to a perfect signal to interference noise ratio (SINR) based power control scheme. Given the required $\operatorname{SINR}\left(\delta_{i}\right)$ for each user, the code division multiple access (CDMA) spreading factor, the bit rate $r_{i}$, the chip rate $C$ of the system (3.84 Mcps), and the required transmit power of station $j$ to satisfy QoS of user $i$ are given by [18]

$$
\begin{aligned}
& p_{i j} \\
& =\delta_{i} v \frac{r_{i}}{C}\left[(1-\omega) P_{j} l_{i j} G_{j}+\sum_{k \in U \backslash \mathrm{HN}_{j}, k \neq j} l_{i k} G_{k} \bar{p}_{k}+n\right] \frac{1}{l_{i j} G_{j}},
\end{aligned}
$$

where $l$ is the path loss, $G$ represents the antenna gain, $n$ is the thermal noise, and $P$ is the transmit power. This process works in an iterative way taking into account power allocations in other cells explicitly. The iterative process is continued until convergence is achieved. The transmit power converges to the minimum possible value for all BSs [18]. The mean transmit power of the base station is given as the summation of the control and the traffic channels:

$$
\bar{p}_{j}=\sum_{i \in \mathrm{CV}_{j}} v_{i} p_{i j}+p_{j}^{c} \leq P_{j} .
$$

\subsection{Base Station Characteristics}

3.5.1. Power Consumption of Base Stations. An empirical model $[22,23,30]$ describing a traffic proportional power consumption characteristic of BS was used:

$$
\begin{gathered}
W_{j}=b \cdot \bar{p}_{j}+c, \quad j \in S, \\
\text { Macro }: \longrightarrow b_{\mathrm{Ma}}=22.6, \quad c_{\mathrm{Ma}}=412.4 \mathrm{~W}, \\
\text { Micro }: \longrightarrow b_{\mathrm{Mi}}=5.5, \quad c_{\mathrm{Mi}}=32 \mathrm{~W} .
\end{gathered}
$$

The FAPs are residential stations with low power consumption characteristics which are independent of the number of users served. For the purpose of our investigation, it was assumed that [31]

$$
W_{f}^{F}=10 \mathrm{~W}, \quad f \in F .
$$

3.5.2. Capacity of Base Stations. The number of users that can be served by a BS depends on the available resources. These are mainly related to transmit power limitations and data rates that can be processed by the IT equipment. The used capacity constraints are

$$
\begin{gathered}
\operatorname{Load}_{j}=\frac{\bar{p}_{j}}{P_{j}} \leq 1, \quad j \in S, \\
\sum_{i \in \mathrm{CV}_{j}} x_{i j} r_{i} \leq R_{j}, \quad j \in S, \\
\sum_{i \in \mathrm{CV}_{j}} x_{i j} \leq 4, \quad j \in f .
\end{gathered}
$$

In the above equation, $R_{j}=14 \mathrm{Mbps}, j \in M_{a}, R_{j}=4 \mathrm{Mbps}$, $j \in M_{i}$, and $r_{i}$ is the bit rate of user $i$. For the FAPs it was assumed that they can serve 4 users simultaneously [27].

3.6. Traffic Profile. The examined traffic profile is presented in Figure 2 [32]. During low traffic hours, met at around 3 am, the normalized traffic is approximately $15 \%$ of the maximum. From the traffic curve it can be observed that, on average, the network is approximately $57 \%$ occupied and that most of the time the network operates below 57\%. This means that underutilization of the available resources is met. This indicates that serious underutilization occurs and energyaware BS management schemes need to be put into place to save energy.

\section{Network Architectures}

According to the operator's objectives in terms of CAPEX and OPEX, different network planning strategies can be followed. For the purpose of our investigation three different network topologies were considered. Since the network planning is proven to be nondeterministic hard (NP-hard), the network topologies were derived by a genetic algorithm (GA) optimization technique. In particular, we consider the minimization of the number of BSs to provide a predefined QoS over the area (named as TX strategy), the minimization 


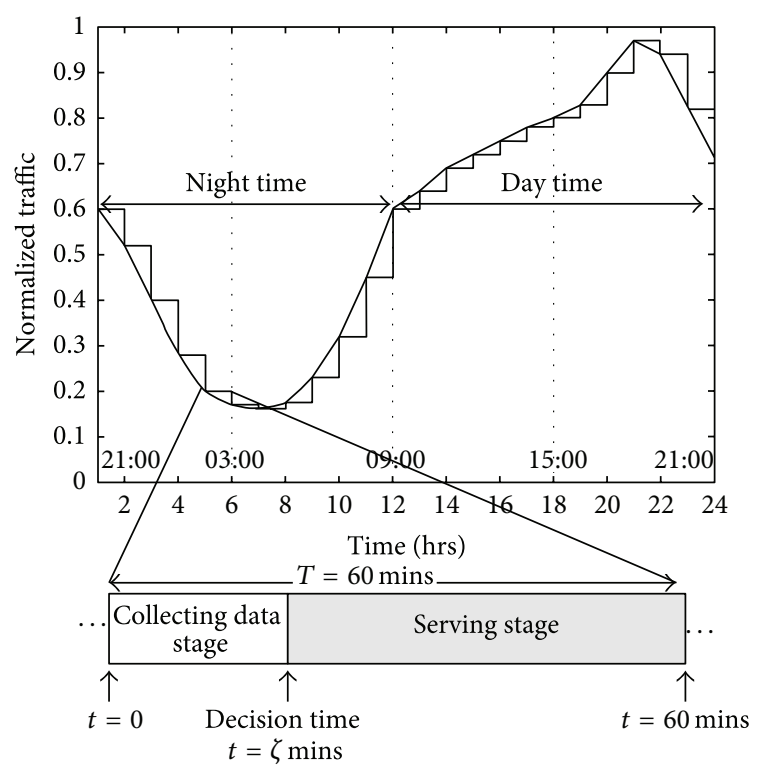

Figure 2: Traffic profile and pseudo real time management procedure.

of power consumption of the network for the same QoS (named as MP strategy), and finally a hybrid approximation (named as HY strategy) as shown in [18]. The GA was used to search the decision variables of the problem that are the base station positions, $a_{j}$ (a binary indicator indicating if a BS exists at a given location), the type of the station, $T_{j}$ (binary indicator indicating macro- or micro-BS), and the transmit RF power, $P_{j}$ [18]. The radio planning procedure was implemented for two traffic scenarios. The first planning is performed considering the peak traffic periods while the second one is applied to low traffic periods. The final network topology comprises a set of BSs $D \subset S$.

Three sets of BSs are distinguished in the network. Set $\mathrm{MD} \subset D$ is the critical base stations that cannot be set to sleep mode, but it participates in the on-/off-management game by providing load information and by coordinating a subset of BSs. Set FD $\subset D$ of BSs can change its state of operation and is named as flexible stations, and finally set $\Omega_{k} \subset \mathrm{FD}, k$ is the index, is the set of BSs that is controlled in the pseudo distributed algorithm by a critical BS, $k \in \mathrm{MD}$. The set $\Omega_{k}$ is defined according to the cell overlap parameters of the cells. By indicating $\Psi_{k j}$ with $0 \leq \Psi_{k j} \leq 1$, the normalized cell overlap value is between stations $k \in \mathrm{MD}$ and $j \in \mathrm{FD}$ and then set $\Omega_{k}$ is defined as

$$
\Omega_{k \in \mathrm{MD}}=\{j\} \longrightarrow \underset{j \in \mathrm{FD}}{\arg \max }\left(\Psi_{k j}\right) .
$$

The aim of the two planning phases is to define the set of flexible BSs for each network configuration. It must be noted that FAPs are not included within the radio planning procedure since they are not managed by the operator. The examined network configurations are shown in Table 1.

4.1. High Traffic Planning. Following the definitions presented in Section 3.1, let $U^{\max }$ be the set of users during
TABLE 2: Set of deployed and managed base stations.

\begin{tabular}{lccc}
\hline Planning & Set & Number of macros & Number of micros \\
\hline \multirow{3}{*}{ TX } & High traffic & 5 & 1 \\
& Low traffic & 2 & - \\
& Flexible stations & 3 & 1 \\
\hline \multirow{3}{*}{ MP } & High traffic & 1 & 14 \\
& Low traffic & 1 & 7 \\
& Flexible stations & - & 7 \\
\hline \multirow{3}{*}{ HY } & High traffic & 4 & 6 \\
& Low traffic & 2 & 3 \\
& Flexible stations & 2 & 3 \\
\hline
\end{tabular}

TABLE 3: Yearly saving (\%) under different strategies.

\begin{tabular}{lccc}
\hline & TX & MP & HY \\
\hline No femto & 12.6 & 8.8 & 22.4 \\
25 no femto priority & 15.0 & 11.0 & 25.9 \\
25 femto priority & 25.2 & 14.0 & 31.0 \\
75 no femto priority & 19.4 & 15.4 & 32.1 \\
75 femto priority & 43.6 & 22.0 & 42.0 \\
\hline
\end{tabular}

peak hour, with cardinality $N^{\max }$. The high traffic planning is performed to fulfill coverage and capacity issues for the maximum possible number of users.

4.1.1. Minimum Transmitter (TX). The TX strategy minimizes the number of BSs and the objective function can be written as

$$
\mathrm{TX} \longrightarrow \min \sum_{j \in S} a_{j} .
$$

The final network configuration comprises BSs $j \in D^{\mathrm{TX}}$. This network configuration yields to a network with a small number of high power BSs (mainly macros; Table 2).

4.1.2. Minimum Power Consumption (MP). This strategy corresponds to a network configuration with the minimum power demands. The objective is

$$
\mathrm{MP} \longrightarrow \min \sum_{j \in S} W_{j}
$$

The decision variables and constraints are the same with the TX strategy. The final network configuration is described by $j \in D^{\mathrm{MP}}$. This network configuration yields to a network comprising a large number of low power base stations (mainly micros; Table 2).

4.1.3. Hybrid Scenario (HY). Hybrid network is the topology that falls in between the TX and MP. Thus there are a smaller number of BSs compared to TX and higher power consumption compared to MP. The HY network configuration is a balanced macro-/micronetwork that is closer to real network deployments. The final network configuration is described by $j \in D^{\mathrm{HY}}$ (see Table 3). A more detailed description of the HY strategy is given in [18]. 

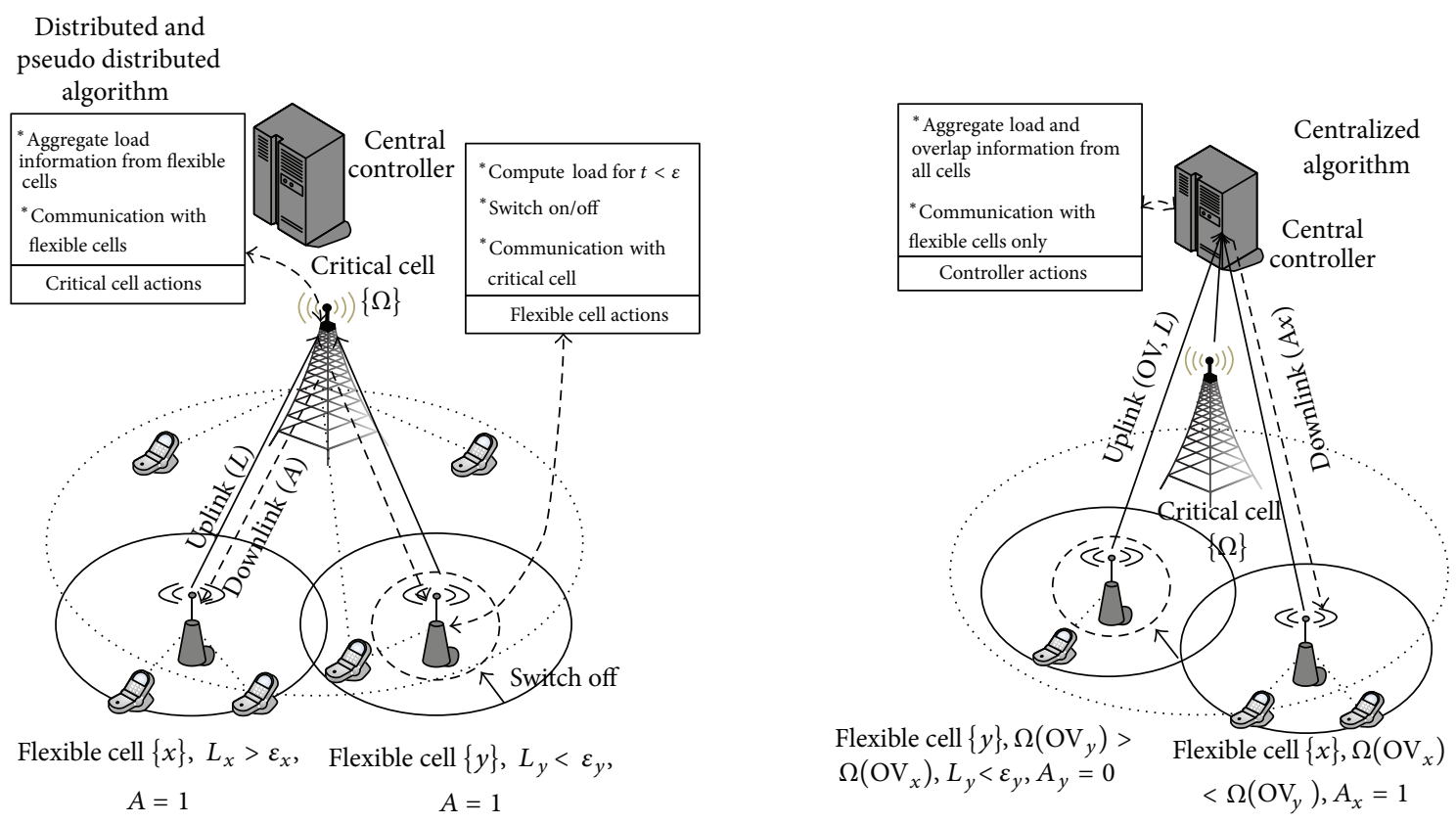

FIGURE 3: Base station management architectures.

4.2. Low Traffic Planning. Most of the BSs at low traffic periods are underutilized and the operation of NCPI equipment reduces the energy efficiency of the network and increases the energy waste. To define the set of flexible BSs, we perform a minimum transmitter optimization strategy for each configuration $D^{X}$. It is similar to the minimum transmitter planning strategy, but the optimization algorithm searches the state space of $D^{X}$ instead of the initial population of possible base station locations $(S)$ and provides the topology $\operatorname{MD}^{X} \subset D^{X}$ that satisfies QoS and coverage for the minimum number of users $U^{\mathrm{min}}$. The objective function for that case is

$$
\mathrm{MD}^{X} \longrightarrow \min \sum_{j \in D^{X}} a_{j} .
$$

All constraints remain the same. For the off-peak planning phase, the optimization algorithm searches only the $a_{j}$ variables whereas the type of BSs $T_{j}$ and the maximum transmit power $P_{j}$ remain the same as in $D^{X}$.

The set of flexible BSs, $\mathrm{FD}^{X}$, is described as $\mathrm{FD}^{X}=$ $D^{X} \backslash \mathrm{MD}^{X}$. These stations model the degrees of freedom of the mobile network operator during the on-/off-management scheme.

\section{Base Station Management Schemes}

Base station management schemes can be performed in a centralized, distributed, or pseudo distributed manner. The control schemes present differences in terms of performance and complexity and are implemented by the Operation, Administration, and Management (OAM) part of the network $[3,8,13,14]$.
5.1. Centralized Management. This scheme is performed by the central controller of the network and decision is taken from OAM (Figure 3, right plot). The controller falls within the administrative domain of the mobile operator and captures critical data of the network. Two centralized approximations are investigated in this paper and these are the least load (LL) and the most overlap (OV) algorithms [8]. All heuristics start by considering a topology in which all BSs are powered on. Then the algorithm checks iteratively if a given BS can be turned off. At each iteration, the considered BS is removed from the topology. User coverage and BS capacity are then recomputed on the residual topology. If both coverage and capacity are still fulfilled, then the selected BS is definitively powered off. Algorithm 1 reports a schematic description of the heuristics. The complexity of the centralized scheme is equal to $O(\overline{\bar{D}})$ where $D$ is the set of BSs of the network.

The least load (LL) strategy is based on the load sustained by the BSs in the considered deployment. Specifically, the BSs are selected starting from the least loaded one. The rationale is that low loaded BSs are more likely to be switched off first, avoiding frequent off-/on- transitions. During the LL algorithm the flexible BSs report to the controller their load and the controller decides if they can be switched off. A binary command $A=\{0,1\}$ is used to describe if a BS should switch off $(A=0)$ or on $(A=1)$ according to coverage and capacity issues.

The most overlap (OV) strategy takes instead into account the overlapping coverage areas existing among neighboring BSs. The intuition is that, in dense deployments, several BSs are necessary to provide capacity during the peak hours, but they are redundant during low traffic periods. In this case, 


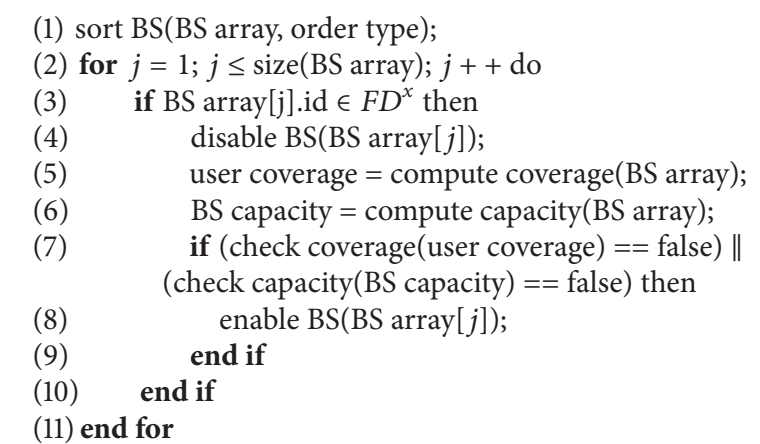

Algorithm 1: Centralized management schemes.

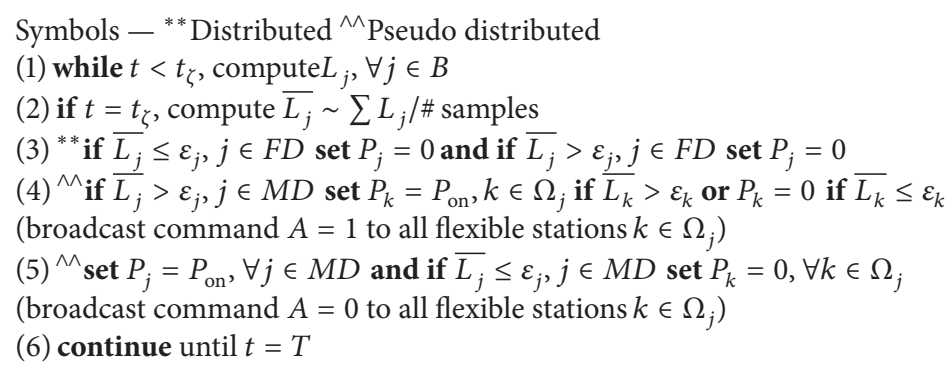

Algorithm 2: Distributed and pseudo distributed management schemes.

the BSs are sorted according to decreasing overlapping. For each BS, the overlapping is computed as the number of active users that can hear the current BS and at least another BS over the total number of users that can hear the current BS. We say that a user can hear a BS if the SINR threshold requirement on the control channel is satisfied.

5.2. Pseudo Distributed Management. The pseudo distributed algorithm authorizes critical BSs to initiate on-/off-command flow to the flexible stations that fall within their administrative domain. Switch-on/-off commands are given hierarchically by stations $k \in \mathrm{MD}$ to flexible stations $j \in \Omega_{k}$ to maintain a smooth QoS during transitions. In this approach, the network is divided into distinct administrative clusters and communication overheads are only limited within each cluster (Figure 3, left plot). Each flexible station reports in the uplink the instantaneous load. The critical station aggregates data and it computes its own load. Decision parameter $A$ (binary parameter $A=\{0,1\}$ ) is broadcasted to all flexible stations $j \in \Omega_{k} . A=1$ means that the flexible stations are allowed to change their state, whereas $A=0$ means that all flexible stations in the administrative domain of the critical station should switch off. The pseudo code of this scheme is given in Algorithm 2. The complexity of the algorithm within each cluster $k$ is $O\left(\overline{\overline{\Omega_{k}}}\right)$.

5.3. Distributed Management. In the distributed approach, the flexible stations $j \in \mathrm{FD}$ decide locally when to change their state of operation neglecting the traffic conditions in other cells of the network. In this approach, communication overhead between the base stations is minimized. The pseudo code of this scheme is given in Algorithm 2. The complexity for each BS is equal to $O(1)$. The algorithm is similar to the pseudo distributed management of Figure 3, but the decision of the state of each flexible BS is independent of the downlink parameter $A$ of the critical stations. Thus, uplink and downlink communication between critical and flexible stations are minimized.

5.4. Algorithm Implementation. The BS management schemes adapt the network according to instantaneous load conditions. The flexible stations can change their state of operation based on pseudo real time network conditions. Pseudo real time conditions indicate that decisions are made at every predefined period of time, $T$, which can be defined by the mobile operator. To help the flexible stations to decide in which mode they will operate, we set a load threshold $\varepsilon_{j}$, $\forall j \in \mathrm{FD}$.

The concept of the proposed management algorithms is presented in Figure 2. A typical day, DT, is quantized into $X$ periods with $X=\mathrm{DT} / T$. Each period $X$ contains two stages: the collecting data stage for $0<t<t_{\zeta}$ and the serving stage for $t_{\zeta}<t<T$. Within the collecting data stage flexible stations serve the users or they can only gather samples of traffic load to calculate the mean traffic load. The BSs might change their mode at a specific point in time $\left(t_{\zeta} \sim 5 \mathrm{mins}\right)$. 
During the serving stage, the remaining active BSs operate to serve the users. The management algorithms guarantee a minimum outage probability $p_{0}$ for the active users $M(t)$.

5.5. Correlation to Recent Standards. According to 3GPP, the BS can operate into three distinct states to support the switch-on/-off scheme [3]. The no-ES (ES refers to energy saving) state is during high traffic where the BS cannot be set in sleep mode. The ESaving state is the switch-off state of the BS. The EScompensate state is the state where the BS is on to support coverage of nearby switched off BSs. Based on our categorization, critical stations are always in no-ES state or equivalently in EScompensate and flexible stations during low traffic periods can be at ESaving. 3GPP presents three control schemes. Centralized and distributed schemes are similar to the schemes described above. For the centralized case the OAM of the network initiates energy saving operation and determines the BS to be set in sleep mode. In the distributed case the OAM initiates energy saving operation of the network, but each BS decides on the state of operation (on/off) independently. Finally, a hybrid scheme is also described in [3] and it presents crucial differences to the proposed pseudo distributed case. In the 3GPP hybrid management, the BS switch-on/-off is implemented in collaboration with the OAM and the individual BSs targeting global optimization. The examined pseudo distributed case is related to a clustering solution that defines smaller administrative domains for BS management.

\section{Modeling Energy Efficiency}

6.1. Mobile Network Operator Power Consumption. This metric computes the network power consumption related to the administrative domain of the mobile operator (macro- and micro-BSs). The network power consumption is computed according to the power needs of the macro- and micro-BSs in $(8 \mathrm{a})$ and $(8 \mathrm{~b})$ :

$$
\operatorname{MPC}[W]=\sum_{j \in D} W_{j}=\sum_{j \in S} a_{j} W_{j} .
$$

The energy consumption over a given time window $\Gamma$ is thus computed according to

$$
\operatorname{MEC}[\mathrm{Wh}]=\sum_{j \in S} \int_{0}^{\Gamma} a_{j} W_{j}(t) d t .
$$

6.2. Energy per User. This metric associates the required energy to serve a user in the network. It takes into account the MPC and also the power consumption of the femto stations. The metric is described as

$$
\mathrm{WU}[W / \text { user }]=\frac{\sum_{j \in S}\left[a_{j} W_{j} / \overline{\overline{B_{j}}}\right]+\sum_{f \in F}\left[W_{f}^{F} / \overline{\overline{B_{f}}}\right]}{\sum_{j \in S} a_{j}+\overline{\bar{F}}} .
$$

The metric as presented in (17) is a heuristic and models the average watts per user per station in the network. The first part of the numerator models the watts per user at the administration domain of the mobile network operator (macrocells and microcells), the second part of the numerator gives the watts per user at the administration domain of the femtocell owners, and the denominator creates an average value per station for the whole network.

\section{Simulation Results}

7.1. Femtocell Layer Effect. This section investigates the effect of femtocells on the network performance. It is assumed that the network has 50 FAPs that are randomly placed in the area. The effect of femtocells is examined for the two user-to-femto association rules as presented in Section 3. In the first subplot of Figure 4 the number of users that are associated with the femtocell network is given for the two association rules. It is observed that for the femto priority rule the number of users that are associated with FAP is the same for the three planning strategies and this is expected. For the no-priority case it is observed that for the MP planning strategy a higher number of users are assigned to the FAPs, compared to the other configurations. This is because the MP network configuration comprises a large number of low power microstations that create almost identical channel characteristics with the FAPs. In the lower subplots of Figure 4 the comparison of the power consumption of the network for the no-femto and 50 femto cases is presented. It is observed that the femtolayer can reduce power needs of the network and the effect is more significant for the TX strategy. This is expected since the TX network configuration comprises a small number of high power stations (mainly macros) whose power consumption is proportional to the input load.

The femtocell effect on the energy efficiency metrics of (17) is presented in Figure 5. The figure presents comparison with the case of no femtos used. It is observed that the femto priority degrades the performance of the network for low traffic periods whereas it increases the efficiency during peak hours. On the other hand, the no-priority rule presents opposite characteristics. The efficiency is reduced since the network of the mobile operator serves less number of users but consumes a lot of power due to the no-load losses of the BSs. The effect of the no-load losses upon the energy efficiency is clear for the MP strategy (microstations) where the efficiency does not present significant changes due to the small losses of the microstations. Taking into account that the mean number of active users in the network is approximately 400 , it can be concluded that, for the specific network, the femto priority user association rule is expected to increase the energy efficiency.

7.2. Base Station Management Effect. Figure 6 presents the reduction of the network power needs with respect to the case where no management is imposed, assuming no femtocells. It is observed that the centralized OV strategy yields the highest gains which are almost identical to the centralized LL strategy. The pseudo distributed management scheme is the scheme that presents lower gains compared to the centralized schemes but higher gains compared to the distributed one. This is expected since the critical BSs of 

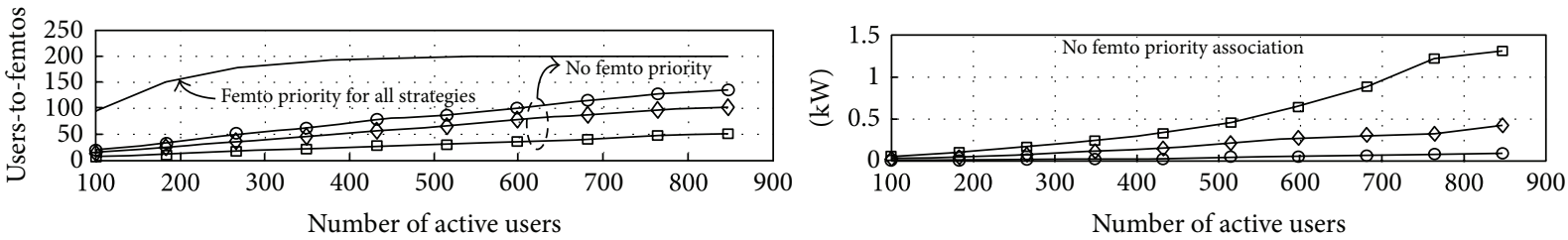

$\diamond \square \mathrm{MPC}_{\text {No_FAP }}-\mathrm{MPC}_{50 \mathrm{FAPS}}$

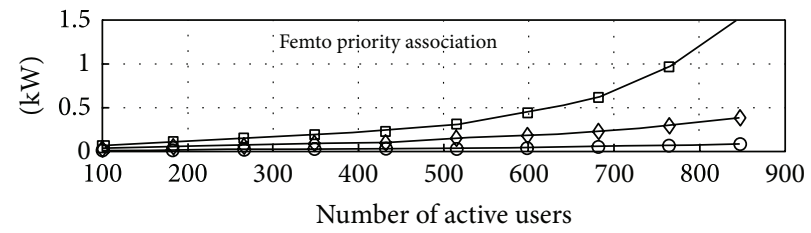
○X strategy
$\diamond$ HY strategy
○ MP strategy

FIGURE 4: The upper subplot presents a number of users that are connected to FAPs for the two association rules. The lower subplots present the network power consumption of the network compared to the no femto case. 50 FAPs randomly positioned in the network were assumed.
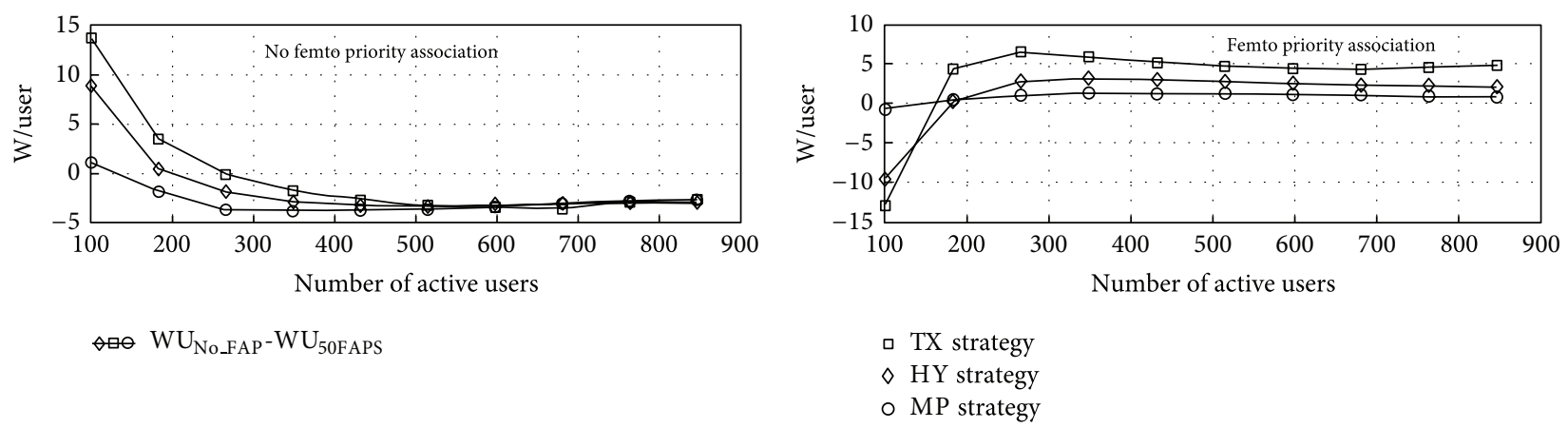

FIGURE 5: Energy efficiency metrics for the femto priority and the no priority cases compared to the no femto scenario. 50 FAPs were assumed.
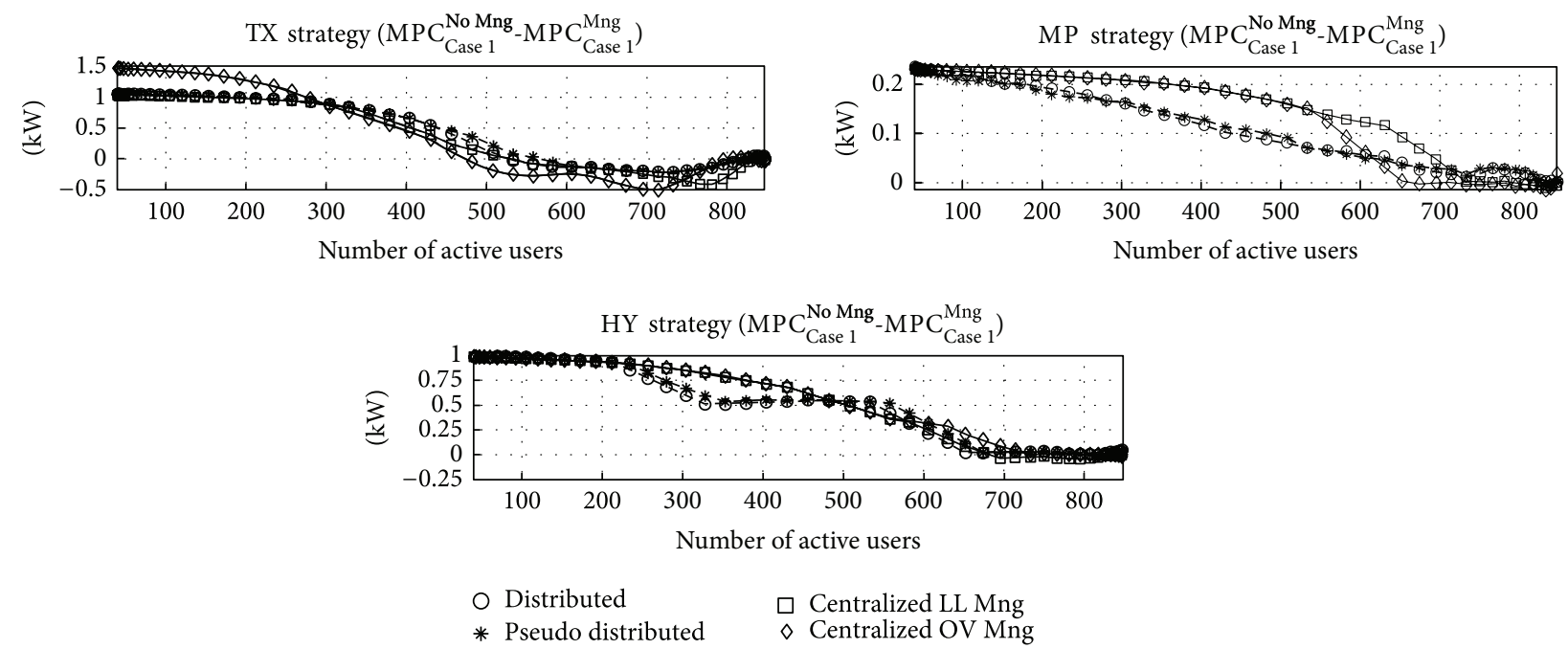

FIGURE 6: Comparison of network power consumption relative to the no management case. 
the network coordinate more efficiently the subset of flexible BSs. It can be observed that for low traffic periods the savings are significant and this is because a large number of BSs are set to sleep mode. On the other hand, during peak traffic the management schemes converge to the performance of the no-management case. During each network transition, the coverage and the outage probability was almost equal to the minimum bounds imposed by the planning phase. The negative values observed for the centralized management indicate that if the network operates with small number of BSs at maximum load can yield worst energy performance compared to no management. This is obvious for 500 and 700 active users of the TX strategy for the centralized OV control. In that case, the network operates with some BSs at sleep mode, meaning that the active BSs are operating near maximum capacity and thus the total consumption is to be higher compared to the case where all BSs are on. Of course, this finding is sensitive to parameters $b$ and $c$ of (8a) and (8b). For the simulation results it was assumed that the load threshold for macrocell stations is equal to $\varepsilon=0.3$ and $\varepsilon=0.03$ for microcell stations [9]. Regarding the outage and network coverage, the centralized algorithm pushed the values to the lowest limits in order to keep the energy efficiency to the maximum value. The distributed and the pseudo distributed algorithms present a better outage and network coverage and a smoother transition between the different states. From the simulation results and by taking into account the increased overhead of the centralized management schemes, the pseudo distributed algorithm was assumed to better reflect real mobile network implementations.

7.3. Base Station Management and Femtocells. This section explores the effect of the femtocell layer on the BS management scheme. The pseudo distributed case is only examined since it was found that it is more appropriate for practical implementations. The scope of the analysis is to identify energy savings and network peak power reduction by increasing the number of femtos in the network. In addition, we investigate the increase of time in which the femtocell layer enables switch off management of BSs in the network as well as the effect on the coverage of the network.

The first simulation result explores the HY planning strategy over the daily traffic profile of Figure 2 . The network power consumption and the number of active BSs for the case of 25 and 75 FAPs are plotted in Figure 7. By increasing the number of FAPs in the network, the BSs management scheme enables more flexible stations to be in sleep mode for larger periods of time with respect to the no femto case. The femtocell layer together with BS management scheme makes the power consumption of the network more proportional to traffic. The femto priority rule guarantees high energy savings, peak power reduction, and larger time periods in which the BSs are set in sleep mode. This is expected since the femtolayer absorbs a higher amount of traffic. The drawback of implementing the femto priority rule is that it is difficult to arrange special agreements between users and mobile operator. In addition, handover mechanisms for moving users are important issues.
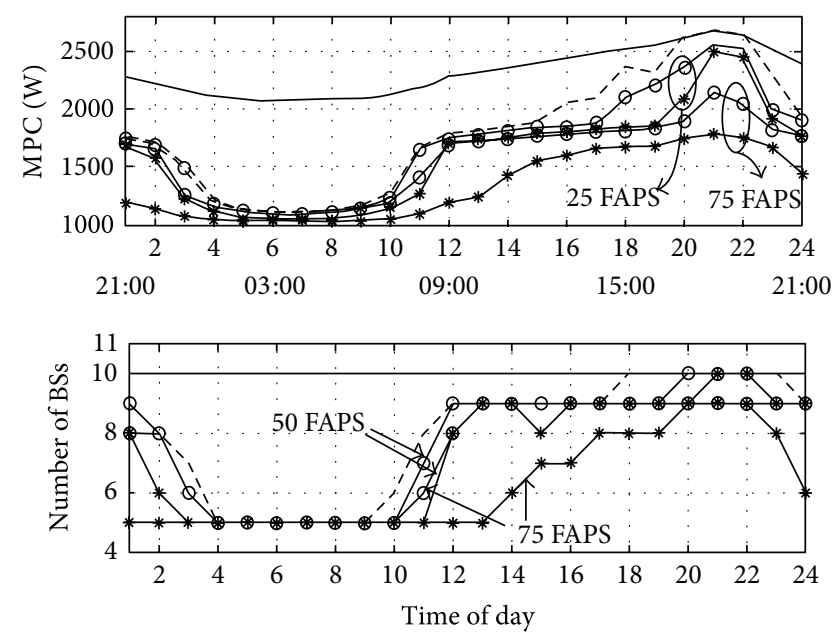

$$
\begin{array}{ll}
\text { O No femto priority } & - \text { No Mng, no femto } \\
* \text { Femto priority } & --- \text { Mng, no femto }
\end{array}
$$

FIGURE 7: Energy consumption and number of active BSs over a typical day for the HY network configuration. Femtolayer effect is presented.

Figure 8 presents the energy savings and the peak power reduction as a function of active FAPs in the network. It can be observed that by increasing the number of FAPs the energy savings (16) are increased in an almost linear function. In addition, the peak power is reduced and this can have a significant effect if one considers the case where the BSs of the network are served by RES with limited power supply, in an island mode (net zero operation). By increasing the number of FAPs, it is also observed that the time interval during a day where BSs are set in sleep mode is significantly increased. This is more obvious for the MP strategy and the femto priority rule where almost the whole day the femtolayer enables more BSs to be set in sleep mode. Finally, it can be concluded that the coverage is improved by increasing the number of femtos. One can investigate similar to the smart grid demand response algorithms to enable the network to adapt its own power curve according to the available RES capacity. It is believed that the femtocell layer will open new business models for cellular networks that are supplied by RES.

7.4. Long-Term Operator Trajectories and Savings. In this section, we first briefly analyze the typical costs that operators face to deploy and maintain a network of BSs; then, we compute the potential savings achievable with the proposed sleep mode schemes. Specifically, we distinguish between operational expenditures (OPEX) and capital expenditures (CAPEX) that vary significantly between macro- and microbase station sites. Indeed, the cost of a macro-BS can be about seven to ten times higher than the one of a micro-BS [33].

In our cost assessment analysis, we use the typical costs for macro- and micro-BSs reported in Tables 1 and 2 of [33]. The CAPEX includes the cost to buy equipment (antennas, 

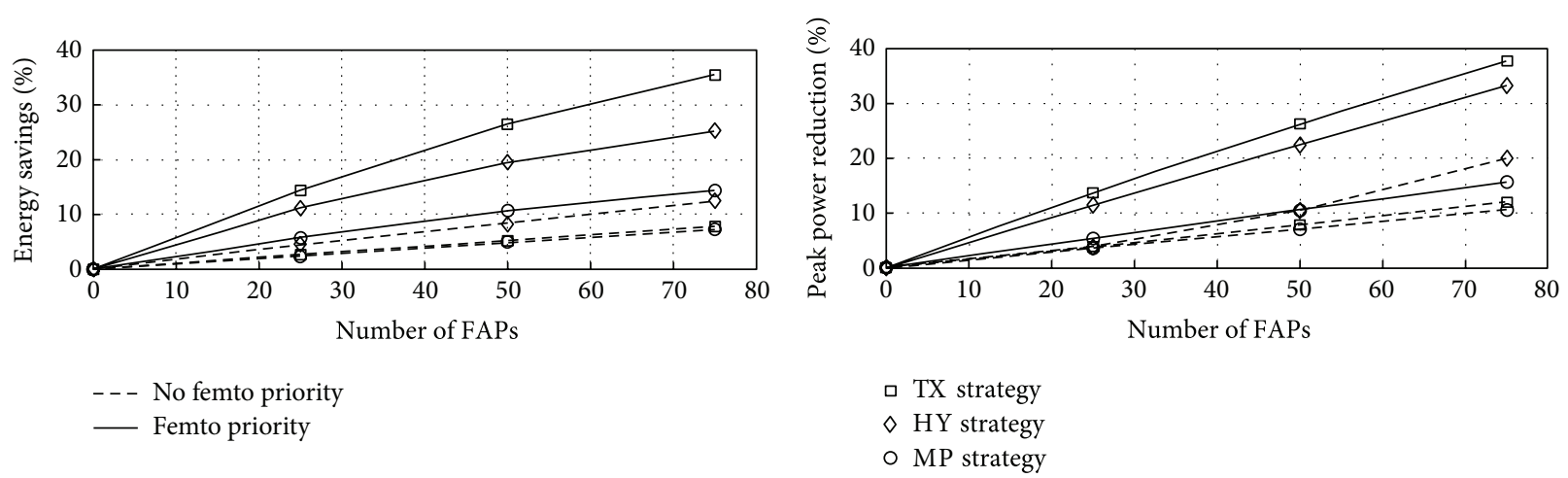

FIGURE 8: Energy savings, peak power reduction, and number of hours that BSs can be switched off and coverage relative to the no femtocell case. Comparisons concern both femto and no femto priority user association rule.

batteries, air conditioning, etc.) and the costs for site acquisition and deployment. The OPEX includes the costs for BS site rent, maintenance, power, and operation. Concerning the electricity cost, we assume that the cost of a KWh is equal to 0.1 Euro [30,34]. Moreover, we can also compute the yearly saving in terms of $\mathrm{tCO}_{2}$ emissions, by imposing that about $500 \mathrm{grCO}_{2}$ are emitted per $1 \mathrm{KWh}$ [34].

In our analysis, first, we indicate which deployment among the ones obtained with the three strategies (TX, MP, and HY) is the best in terms of costs. Then, given the deployment, we compute the total yearly cost of the network for the following cases: (i) all the BSs are always on, and (ii) sleep modes are used. Finally we evaluate the energy that can be saved thanks to BSs' sleep modes.

In the following we show some numerical results in terms of savings obtained considering the three strategies under different network management schemes. First of all, we compute the CAPEX costs of the deployments. The most expensive deployment is the TX one (about $551 \mathrm{~K} €$ ) since it includes more macro-BSs, while the MP costs about $262 \mathrm{~K} €$ and the HY one costs about $498 \mathrm{~K} €$. Thus, the more convenient strategy in terms of CAPEX is the MP one, since it comprises several but small BSs. The main drawback comes from the software perspective since the coordination of a large number of small cells is a complex procedure.

Then, we compute the savings obtained by adopting sleep mode schemes at the BSs. We report the percentage of achievable savings in Table 3, considering the cases in which (i) no femtocells are exploited in the network management scheme; (ii) 25 femtocells are used with/without any priority scheme; (iii) 75 femtocells are used with/without any priority scheme.

Observe that significant savings can be achieved with the TX and HY strategies, while savings are lower for the MP strategy. Moreover, it is interesting to note that savings are higher when the femto priority strategy is used and increase with the number of femtocells exploited by the network.

We also compute the monetary savings that can be achieved by using the different energy management schemes. For example, considering the femto priority scheme with 75 femtocells and the TX strategy, the yearly saving is about $1250 €$. Considering a typical national network with about
50000 BSs for coverage, we can assume that about $20 \%$ of the BSs present cell overlap due to capacity issues in urban environment. Thus, the yearly saving in a national network can be roughly 2,4 millions of Euros. Moreover, if we consider the savings in terms of $\mathrm{CO}_{2}$ emissions, this means a reduction of about $13000 \mathrm{tCO}_{2}$.

Finally, taking into account both CAPEX and OPEX and assuming that sleep modes are adopted at the BSs, we can conclude that (i) in the short term, the planning phase is the most crucial one from the cost point of view, due to the high CAPEX expenses; (ii) in the long term, significant savings can be achieved by adopting sleep modes at the BSs (due to OPEX reduction); (iii) among the different types of planning strategies that we have considered, the MP deployment (i.e., the one with many micro-BSs) is the most convenient one, and still some savings can be achieved with sleep modes at the micro-BSs.

\section{Conclusions}

This paper investigated algorithms and techniques that can be applied on cellular networks and provide traffic proportional power consumption. Three different planning strategies and BS management schemes were used to investigate potential savings in the network. Furthermore, the paper explored the effect of a heterogeneous network (femtocell layer) on BS management schemes by considering two different userto-femto association rules. It was observed that the pseudo distributed management scheme together with femto priority association rule can provide important energy savings in the long run but also dynamic load control of the cellular network that is regarded as a fundamental element of future demand response services. Finally, it was proved that the femtolayer can improve QoS, coverage, and switch-off time intervals in the BS management scheme, providing more degrees of freedom to the mobile operator to adapt the power consumption in real time.

\section{Conflict of Interests}

The authors declare that there is no conflict of interests regarding the publication of this paper. 


\section{Acknowledgments}

This work was funded from the European Union FP7/20072013 under Grant Agreement no. 257740 (Network of Excellence TREND). The authors would like to thank Dr. Anastastios Karousos for the outdoor channel predictions.

\section{References}

[1] W. Stark, H. Wang, A. Worthen, S. Lafortune, and D. Teneketzis, "Low-energy wireless communication network design," IEEE Wireless Communications, vol. 9, no. 4, pp. 60-72, 2002.

[2] W. Van Heddeghem, M. Deruyck, B. Puype et al., "Power consumption in telecommunication networks: overview and reduction strategies," IEEE Communications Magazine, vol. 49, pp. 62-69, 2011.

[3] 3GPP TR 32.826 v2.0.0 (2010-03), Study on Energy Savings Management (ESM), Release 9, 2010.

[4] 3GPP TR 36.902, E-UTRAN, Self-configuration and selfoptimizing network use cases and solutions, (Rel 9), October 2009.

[5] A. P. Bianzino, C. Chaudet, D. Rossi, and J.-L. Rougier, "A survey of green networking research," IEEE Communications Surveys and Tutorials, vol. 14, no. 1, pp. 3-20, 2012.

[6] M. Gupta and S. Singh, "Greening of the Internet," in Proceedings of the Conference on Applications, Technologies, Architectures, and Protocols for Computer Communications (SIGCOMM '03), August 2003.

[7] L. Chiaraviglio, D. Ciullo, M. Meo, and M. A. Marsan, "Energyefficient management of UMTS access networks," in Proceedings of the 21st International Teletraffic Congress (ITC '09), Paris, France, September 2009.

[8] L. Chiaraviglio, D. Ciullo, G. Koutitas, M. Meo, and L. Tassiulas, "Energy-efficient planning and management of cellular networks," in Proceedings of the 9th Annual Conference on Wireless On-Demand Network Systems and Services (WONS '12), pp. 159166, January 2012.

[9] S. Kokkinogenis and G. Koutitas, "Dynamic and static base station management schemes for cellular networks," in Proceedings of the IEEE Global Communications Conference (GLOBECOM '12), Aneheim, Calif, USA, 2012.

[10] K. Samdanis, D. Kutscher, and M. Brunner, "Self-organized energy efficient cellular networks," in Proceedings of the 21st IEEE International Symposium on Personal Indoor and Mobile Radio Communications (PIMRC '10), pp. 1665-1670, September 2010.

[11] C. Peng, S. B. Lee, S. Lu, H. Luo, and H. Li, “Traffic-driven power saving in operational 3G cellular networks," in Proceedings of the 17th Annual International Conference on Mobile Computing and Networking (MobiCom '11), Las Vegas, Nev, USA, September 2011.

[12] E. Oh, B. Krishnamachari, X. Liu, and Z. Niu, "Toward dynamic energy-efficient operation of cellular network infrastructure," IEEE Communications Magazine, vol. 49, no. 6, pp. 56-61, 2011.

[13] Z. Niu, Y. Wu, J. Gong, and Z. Yang, "Cell zooming for cost-efficient green cellular networks," IEEE Communications Magazine, vol. 48, no. 11, pp. 74-79, 2010.

[14] M. Ismail and W. Zhuang, "Network cooperation for energy saving in green radio communications," IEEE Wireless Communications, pp. 76-81, 2011.
[15] M. Ajmone Marsan and M. Meo, "Energy efficient wireless Internet access with cooperative cellular networks," Computer Networks, vol. 55, no. 2, pp. 386-398, 2011.

[16] F. Richter, A. Fehske, P. Marsch, and G. Fettweis, "Traffic demand and energy efficiency in Heterogeneous cellular mobile radio networks," in Proceedings of the International Conference on Wireless Communications (VTC '10), pp. 1-6, May 2010.

[17] K. Dufkova, M. Popovic, R. Khalili, J. V. Le Boudec, M. Bjelica, and L. Kencl, "Energy consumption comparison between macro-micro and public femto deployment in a plausible LTE network," in Proceedings of the 2nd International Conference on Energy-Efficient Computing and Networking, New York, NY, USA, 2011.

[18] G. Koutitas, A. Karousos, and L. Tassiulas, "Deployment strategies and energy efficiency of cellular networks," IEEE Transactions on Wireless Communications, vol. 11, no. 7, pp. 2552-2563, 2012.

[19] J. Lorincz, A. Capone, and D. Begusic, "Impact of service rates and base station switching granularity on energy consumption of cellular networks," EURASIP Journal on Wireless Communications and Networking, vol. 2012, article 342, 2012.

[20] S. Buzzi and D. Saturnino, "A game-theoretic approach to energy-efficient power control and receiver design in cognitive CDMA wireless networks," IEEE Journal on Selected Topics in Signal Processing, vol. 5, no. 1, pp. 137-150, 2011.

[21] O. Arnold, F. Richter, G. Fettweis, and O. Blume, "Power consumption modeling of different base station types in heterogeneous cellular networks," in Proceedings of the Future Network and Mobile Summit, Florence, Italy, June 2010.

[22] A. J. Fehske, F. Richter, and G. P. Fettweis, "Energy efficiency improvements through micro sites in cellular mobile radio networks," in Proceedings of the IEEE Globecom Workshops (Gc Workshops '09), Honolulu, Hawaii, USA, December 2009.

[23] G. Auer, V. Giannini, I. Gódor et al., "Cellular energy efficiency evaluation framework," in Proceedings of the 73rd IEEE Vehicular Technology Conference (VTC '11), May 2011.

[24] C. Khirallah, J. S. Thompson, and H. Rashvand, "Energy and cost impacts of relay and femtocell deployments in long-termevolution advanced," IET Communications, vol. 5, no. 18, pp. 2617-2628, 2011.

[25] A. De Domenico, R. Gupta, and E. Calvanese Strinati, "Dynamic traffic management for green open access femtocell networks," in Proceedings of the 75th IEEE Vehicular Technology Conference (VTC '12), pp. 1-6, May 2012.

[26] H.-F. Gerdees, UMTS Radio Network Planning: Mastering Cell Coupling for Capacity Optimization, Vieweg+Teubner Research, MRC, 2008.

[27] J. Zhang and G. de la Roche, Femtocells: Technologies and Deployment, John Wiley \& Sons, 2010.

[28] J. M. Keenan and A. J. Motley, "Radio coverage in buildings," British Telecom Technology Journal, vol. 8, no. 1, pp. 19-24, 1990.

[29] 3GPP TSG-RAN WG4 \#44-bis, "HNB and HNB-macro propagation models," 3GPP Report, 2007.

[30] J. Lorincz, T. Garma, and G. Petrovic, "Measurements and modelling of base station power consumption under real traffic loads," Sensors, vol. 12, no. 4, pp. 4281-4310, 2012.

[31] I. Haratcherev, C. Balageas, and M. Fiorito, "Low consumption home femto base stations," in Proceedings of the 20th IEEE Personal, Indoor and Mobile Radio Communications Symposium (PIMRC '09), pp. 1-5, September 2009. 
[32] G. Auer, V. Giannini, I. Godor et al., "Cellular energy efficiency evaluation framework," in Proceedings of the 73rd IEEE Vehicular Technology Conference (VTC '11), 2011.

[33] M. Werner, M. Naden, P. Moberg et al., "Cost assessment of radio access network deployments with relay nodes," in Proceedings of the IST Mobile and Wireless Communications Summit (IST '08), May 2008.

[34] International Energy Agency (IEA), " $\mathrm{CO}_{2}$ emissions from fuel combustion: Highlights," Tech. Rep., 2009. 

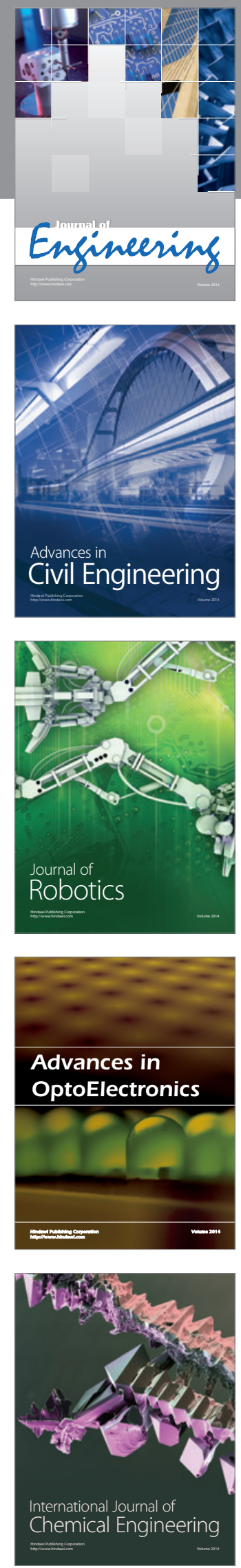

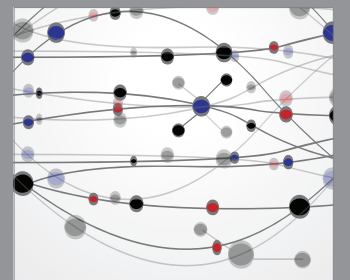

The Scientific World Journal
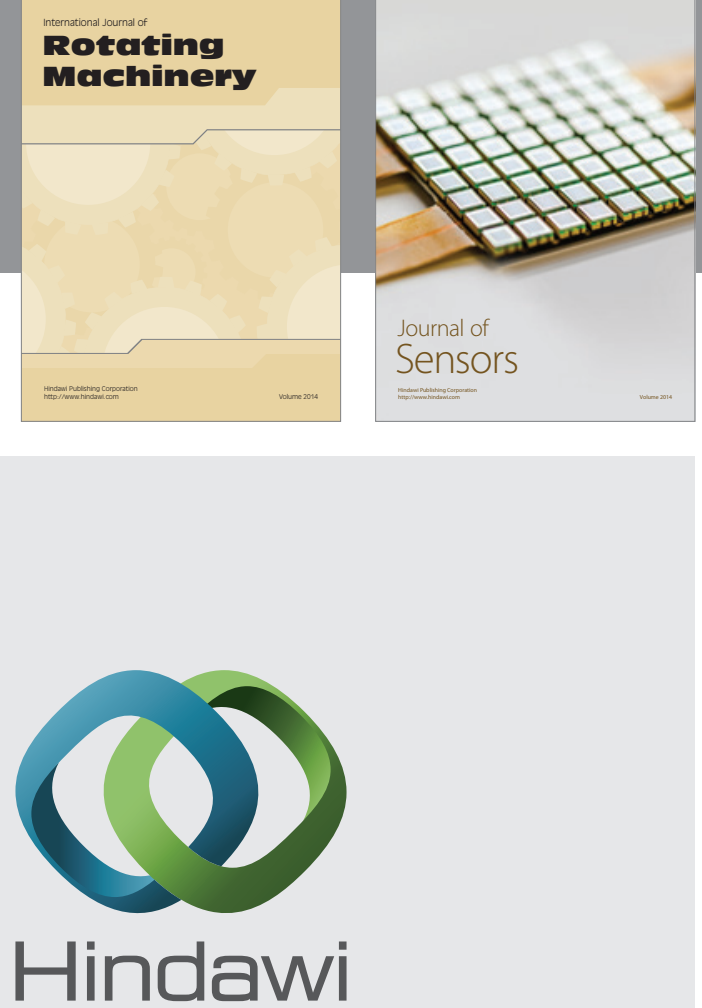

Submit your manuscripts at http://www.hindawi.com
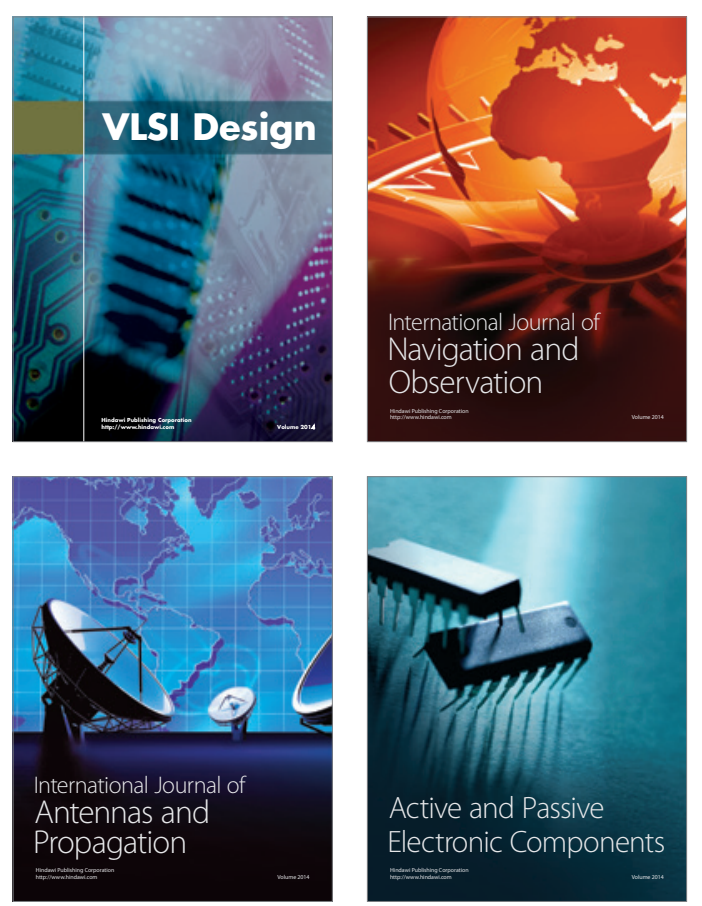
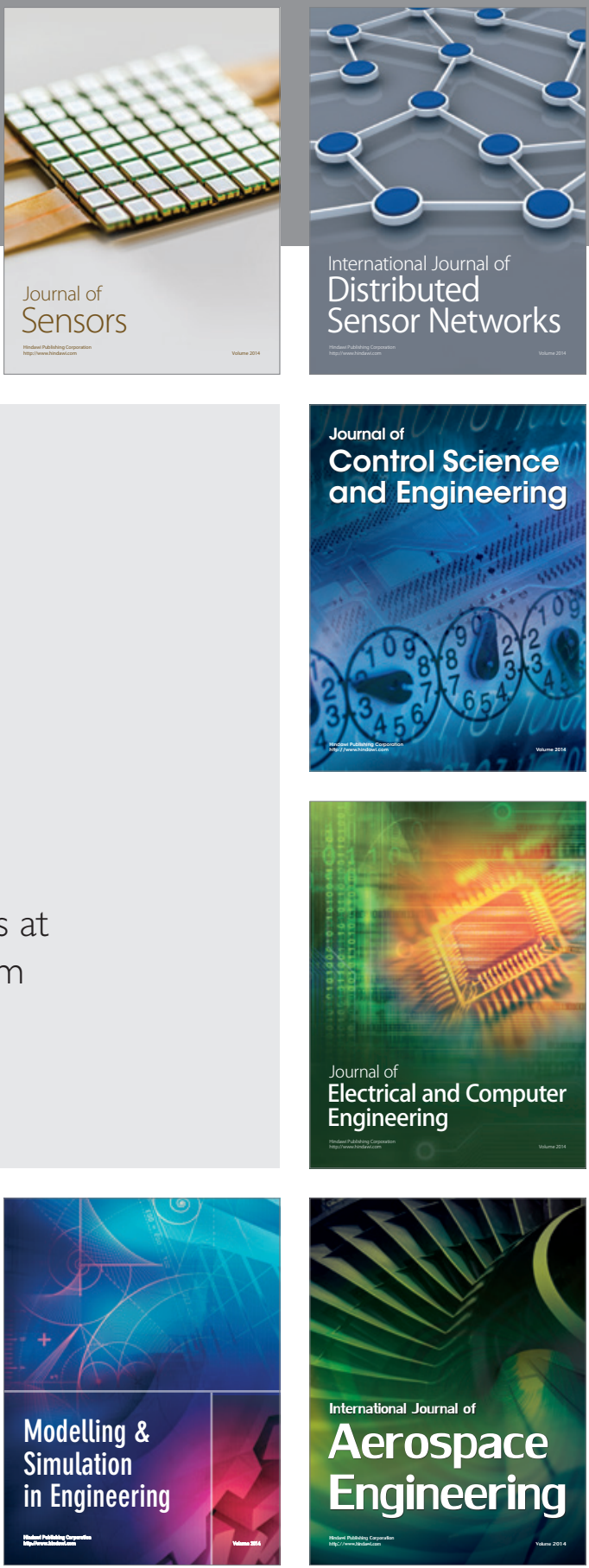

Journal of

Control Science

and Engineering
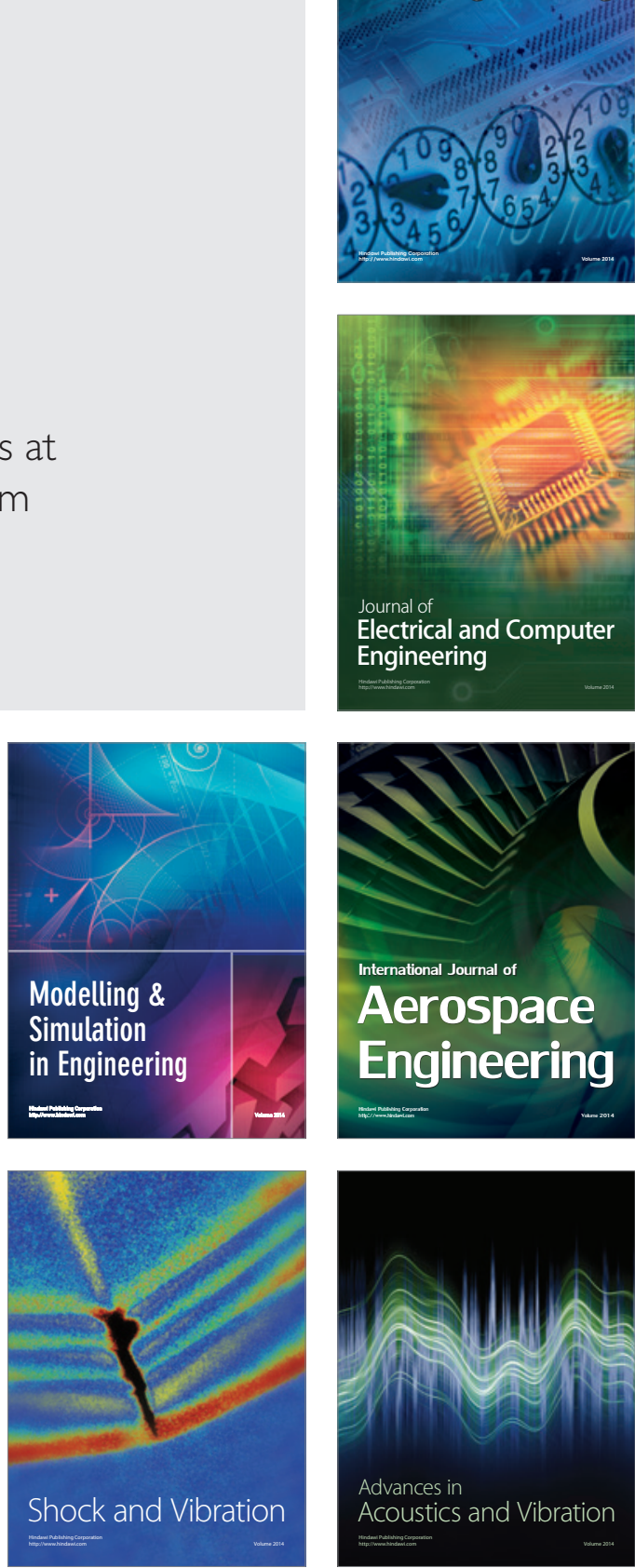